\title{
Synthesis and characterization of 2D nanocarbon synthesized by microwave irradiation
}

\author{
Jayesh Cherusseri ${ }^{1}$ and Kamal K. Kar ${ }^{1,2^{*}}$ \\ ${ }^{1}$ Advanced Nanoengineering Materials Laboratory, Materials Science Programme, Indian Institute of \\ Technology, Kanpur, Uttar Pradesh-208016, India. \\ ${ }^{2}$ Department of Mechanical Engineering, Indian Institute of Technology, Kanpur, Uttar Pradesh- \\ 208016, India. \\ *Email:kamalkk@iitk.ac.in
}

Advancements in the field of nanoscience have opened the doors to variety of carbon nanomaterials (CNMs) such as carbon nanotubes, carbon nanopetals, carbon nanofibers, graphene, etc. [1,2]. These CNMs exhibit unique properties such as large surface area, high electronic conductivity, good thermal, chemical and electrochemical stabilities, etc. Hence they are widely used in advanced applications such as structural, biomedical, electronic, etc.

In the present study, 2D graphitic nanocarbon is synthesized by microwave irradiation technique from natural flake graphite (NFG) precursor. Potassium permanganate- perchloric acid- sulphuric acid based graphite intercalation system was used and 2D graphitic nanocarbon sheets are prepared by microwave irradiation.

Figure 1a shows the SEM image of NFG, which indicates the morphology of NFG is flat and flake-like. The flake-like morphology is appropriate to accommodate oxidant molecules within the layers of graphitic carbon sheets during the intercalation process. After the microwave irradiation, NFG is exfoliated in the direction of $c$-axis.

Figure $1 \mathrm{~b}$ shows the SEM image of $2 \mathrm{D}$ nanocarbon, which indicates that the exfoliation process has enabled the expansion throughout the NFG. The thickness of individual 2D nanocarbon sheet is $\sim 100 \mathrm{~nm}$.

The 2D nanocarbon is characterized by Fourier transform infrared (FTIR) spectroscopy and the corresponding spectra is shown in Figure 1c. In the FTIR spectra, the peaks at 1622, 2924, 2854, 1246, 1127, 1040, and $972 \mathrm{~cm}^{-1}$ are correspond to $\mathrm{O}-\mathrm{H}$ stretching, $\mathrm{C}-\mathrm{H}$ stretching of alkyl chain, $\mathrm{C}-\mathrm{OH}$ stretching, and $\mathrm{C}-\mathrm{O}$ stretching, respectively [2].
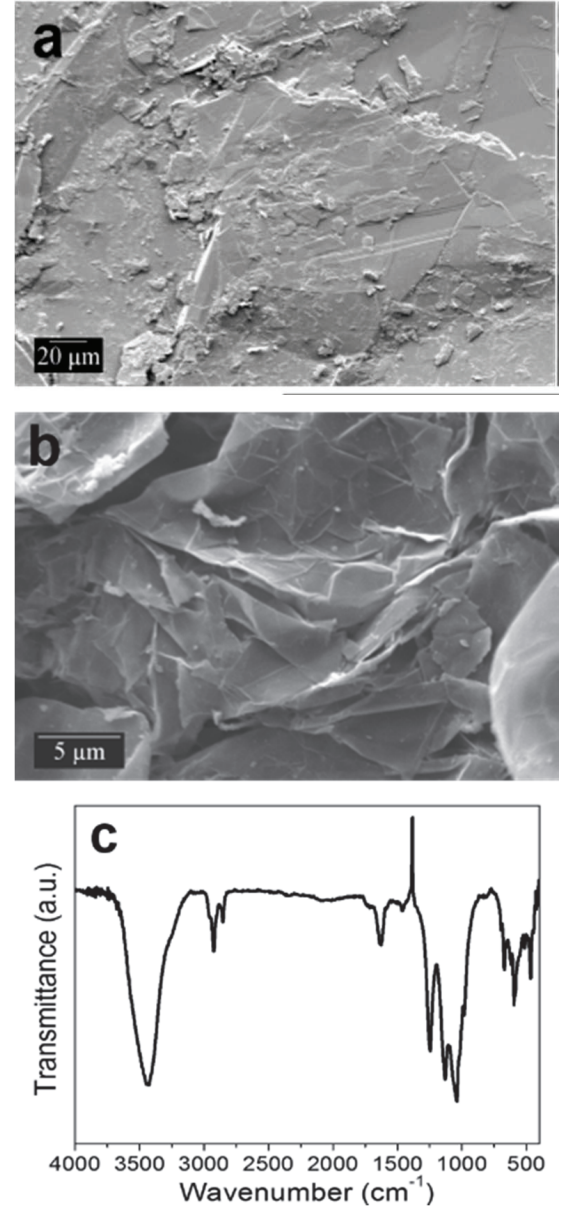

Figure 1: (a-b) SEM images of (a) NFG and (b) 2D nanocarbon; and (c) FTIR spectra of 2D nanocarbon

\section{References}

1. J. Cherusseri, K. K. Kar, RSC Adv. 5 (2015) 34335 .

2. J. Cherusseri, K. K. Kar, J. Mater Chem. A 3 (2015) 21586. 